\title{
Reflectometry-Ellipsometry Reveals Thickness, Growth Rate and Phase Composition in Oxidation of Copper
}

Juan J. Diaz Leon ${ }^{1,2, *}$, David M. Fryauf ${ }^{1,2}$, Robert D. Cormia ${ }^{3}$, Min-Xiam Max Zhang ${ }^{4}$, Kathryn Samuels ${ }^{4}$, R. Stanley Williams ${ }^{4}$, Nobuhiko P. Kobayashi ${ }^{1,2}$

${ }^{1}$ Baskin School of Engineering, University of California Santa Cruz, Santa Cruz, California, 95064, USA

${ }^{2}$ Nanostructured Energy Conversion Technology and Research (NECTAR), Advanced Studies Laboratories, Univ. of California Santa Cruz - NASA Ames Research Center, Moffett Field, California, 94035, USA

${ }^{3}$ Foothill College, Los Altos, California, 94022, USA

${ }^{4}$ Hewlett Packard Labs, Palo Alto, California, 94304, USA

*Electronic mail: jdiazleo@ucsc.edu
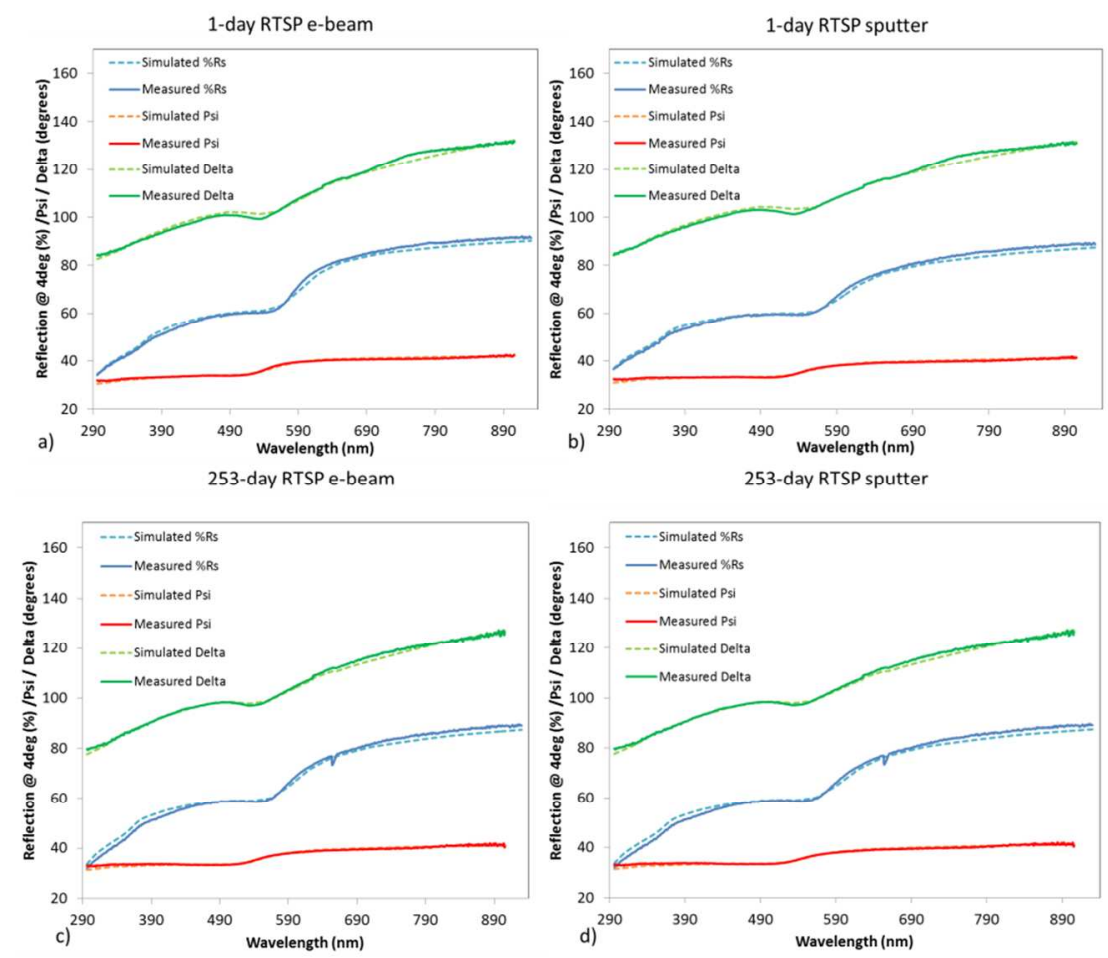

Figure S1. Measured and simulated reflectometry-ellipsometry for the e-beam sample after a) 1 day and b) 253 days of RTSP oxidation time and for the sputter sample after c) one day and d) 253 days of RTSP oxidation time. 


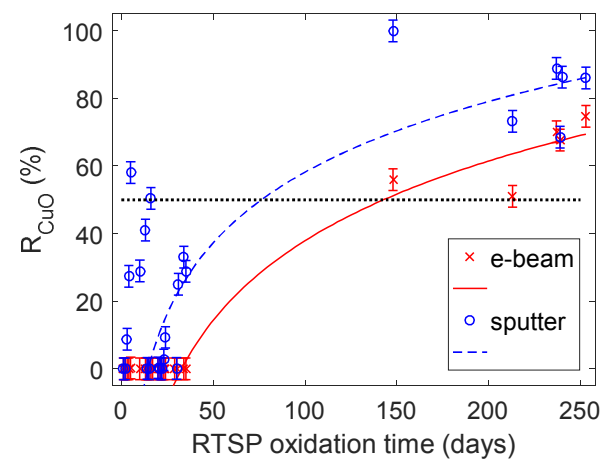

Figure S2. The ratio of the volume fraction of $\mathrm{CuO}$ to the sum of the volume fraction of $\mathrm{CuO}$ and that of $\mathrm{Cu}_{2} \mathrm{O}$, namely $R_{\mathrm{CuO}}$, increases with $\mathrm{RTSP}$ oxidation time. The sputter sample achieves $\mathrm{R}_{\mathrm{CuO}}=50 \%$ before the e-beam sample.

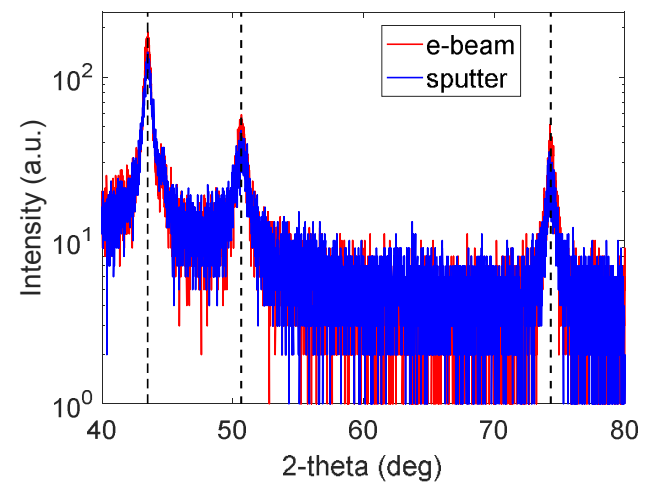

Figure S3. x-ray diffraction (XRD) shows different crystallite size on e-beam and sputtered copper films. 